\title{
Lack of association between right-to-left shunt and cerebral ischemia after adjustment for gender and age Holger Poppert*1, Melanie Morschhaeuser ${ }^{1}$, Regina Feurer ${ }^{1}$, Angelina Bockelbrink ${ }^{2}$, Jens Schwarze ${ }^{3}$, Lorena Esposito ${ }^{1}$, Peter Heider ${ }^{4}$, Dirk Sander ${ }^{5}$ and Bernhard Hemmer ${ }^{1}$
}

\begin{abstract}
Address: ${ }^{1}$ Department of Neurology, Klinikum Rechts der Isar, Technische Universitaet Muenchen, Ismaningerstr. 22, 81675 Muenchen, Germany, ${ }^{2}$ Department of Social Medicine, Epidemiology and Health Economics, Charité - University Medicine Berlin, Luisenstr. 57, 10117 Berlin, Germany, ${ }^{3}$ Department of Neurology, Klinikum Chemnitz, Flemmingstraße 2, 09116 Chemnitz, Germany, ${ }^{4}$ Department of Vascular Surgery, Klinikum Rechts der Isar, Technische Universitaet Muenchen Ismaninger Str. 22, 81675 Muenchen, Germany and ${ }^{5}$ Neurologische Klinik Medical Park Loipl, Thanngasse 15, 83483 Bischofswiesen, Germany
\end{abstract}

Email: Holger Poppert* - poppert@neurovasc.de; Melanie Morschhaeuser - melanie.morschhaeuser@gmail.com; Regina Feurer - feurer@neurovasc.de; Angelina Bockelbrink - Bockelbrink@charite.de; Jens Schwarze - jens.schwarze@skc.de; Lorena Esposito - esposito@neurovasc.de; Peter Heider - heiderpeter@t-online.de; Dirk Sander - d.sander@mac.com; Bernhard Hemmer - hemmer@lrz.tu-muenchen.de

* Corresponding author

Published: 13 October 2008

Journal of Negative Results in BioMedicine 2008, 7:7 doi:10.1 I86/1477-575I-7-7

This article is available from: http://www.jnrbm.com/content/7/I/7

(c) 2008 Poppert et al; licensee BioMed Central Ltd.

This is an Open Access article distributed under the terms of the Creative Commons Attribution License (http://creativecommons.org/licenses/by/2.0), which permits unrestricted use, distribution, and reproduction in any medium, provided the original work is properly cited.

\begin{abstract}
Introduction: A number of studies has addressed the possible association between patent foramen ovale (PFO) and stroke. However, the role of PFO in the pathogenesis of cerebral ischemia has remained controversial and most studies did not analyze patient subgroups stratified for gender, age and origin of stroke.
\end{abstract}

Methods: To address the role of PFO for the occurrence of cerebral ischemia, we investigated the prevalence of right-to-left shunt in a large group of patients with acute stroke or TIA. 763 consecutive patients admitted to our hospital with cerebral ischemia were analyzed. All patients were screened for the presence of PFO by contrast-enhanced transcranial Doppler sonography at rest and during Valsalva maneuver. Subgroup analyses were performed in patients stratified for gender, age and origin of stroke.

Results: A right-to-left shunt was detected in $140(28 \%)$ male and in II 4 (42\%) female patients during Valsalva maneuver, and in $66(13 \%)$ and $44(16 \%)$ at rest respectively. Patients with right-toleft shunt were younger than those without $(P<0.00 \mathrm{I})$. PFO was associated with stroke of unknown origin in male $(P=0.00 \mathrm{I})$ but not female patients $(P>0.05)$. After adjusting for age no significant association between PFO and stroke of unknown origin was found in either group.

Conclusion: Our findings argue against paradoxical embolization as a major cause of cerebral ischemia in patients with right-to-left shunt. Our data demonstrate substantial gender-and agerelated differences that should be taken into account in future studies. 


\section{Introduction}

Despite numerous studies published in the past two decades, the role of patent foramen ovale (PFO) as a risk factor of stroke remains a matter of debate. A significant correlation between PFO and cryptogenic stroke has repeatedly been shown. However, most studies included only small numbers of patients and thus did not allow to adjust the analysis for gender and age.

The aim of our study was to re-evaluate the association between right-to-left shunts (RLS) and stroke subtypes in a patient community large enough to allow multivariate analysis with special consideration of gender-related differences.

\section{Methods \\ Subjects}

The records of 973 consecutive patients examined between January 1997 and December 2005 at the Neurovascular Laboratory of the Department of Neurology at the Klinikum Rechts der Isar, Technische Universitaet Muenchen, were retrospectively reviewed. 210 patients without definite diagnosis of cerebral ischemia and those with artificial heart valves were excluded.

Complete clinical neurological examination, electrocardiogram, and sonographic examination of the extra- and intracranial arteries were carried out in all patients, as well as a cerebral CT or MRI examination, or both. Echocardiography was performed in 683 patients (89.5\%). A 4-lead 24-hour ECG was performed routinely.

All baseline ischemic events were classified according to the TOAST criteria using all diagnostic data available, [1] with one modification: Strokes with conflicting mechanisms were subsumed under "other etiology" instead of classifying them as cryptogenic. Therefore the latter subgroup truly represented strokes without any identifiable etiology.

The TOAST subtyping was performed by one physician (H.P.) who was blinded for the results of the TCD testing.

\section{C-TCD Methodology}

For microembolic monitoring, a 2-MHz pulsed-wave transcranial Doppler device (MULTI-DOP, DWL Elektronische Systeme, Sipplingen, Germany) was used for simultaneous insonation of both middle cerebral arteries (MCA) using simultaneous 64-point FFT and bigate technique. An intensity threshold of $=11 \mathrm{~dB}$ and a time window of 20 seconds after the start of the injection of galactose $\left(\right.$ Echovist $\left.^{\boxplus}\right)$ were chosen.

The patient was placed supine. The transducer was fixed in position with the use of a standard headset. The embolic signals were recorded after bolus injection of galactose $\left(\right.$ Echovist $^{\circledR}$ ) via the right antecubital vein followed by a flush injection of $5 \mathrm{~mL}$ of normal saline. Five seconds after start of the injection, patients had to perform a Valsalva maneuver. This was monitored by means of a pressure gauge, which was connected to a flexible tube with a snorkel mouthpiece. The patients were asked to maintain a pressure of $4000 \mathrm{~Pa}$ (40 mbar) for 5 seconds. Simultaneous monitoring of the Doppler spectrum allowed us to demonstrate an increased intrathoracic pressure as shown by a reduction in the mean velocity in the MCA of at least $25 \%$. In case of a positive finding, the examination was repeated at rest to discriminate functional large versus small shunts.

C-TCD was performed in every case according to our previously published protocol.[2] Except for the choice of contrast medium, our protocol conforms to the Consensus conference of Venice.[3] To ensure a maximum degree of standardization, we used commercially available galactose instead of agitated saline.

\section{Statistical Analysis}

Continuous data are shown as mean and standard deviation (SD); categorical variables are expressed as absolute and relative frequencies. Differences were tested by chisquare and Mann-Whitney U-test as adequate. Associations between PFO, confounding factors, and different subtypes of stroke were calculated by logistic regression analysis and described using odds ratios (OR) with 95\% confidence intervals (CI). The analyses were carried out on the dataset stratified by gender.

All calculations were performed using SPSS 13.0 software (SPSS Inc., Chicago, IL, USA).

\section{Results}

\section{Study Population}

Basic characteristics are given in Table 1.

\section{Detection of embolic signals (ES)}

RLS was detected in $140(28 \%)$ male and in $114(42 \%)$ female patients during Valsalva maneuver $(P<0.001) .66$ (13\%) male and 44 (16\%) female patients showed RLS at rest. Both male and female ES-positive patients were younger $(P<0.001)$ and had fewer traditional vascular risk factors than participants of the same gender without RLS $(P<0.01)$.

In male patients presence of RLS was significantly associated with stroke of unknown origin, whereas in female patients the association did not reach significance (Table 2 ). No stronger association was found in either men or women for the different stroke subtypes and ES at rest (Table 3). 
Table I: Basic characteristics of the study population

\begin{tabular}{|c|c|c|c|}
\hline & Overall Study Population $(n=763)$ & Male Patients $(n=494)$ & Female Patients $(n=269)$ \\
\hline Age, y, mean (SD) & $58.2(14.7)$ & $59.8(13.7)$ & $55.2(16.0)$ \\
\hline Hypertension, n (\%) & $462(61)$ & $323(65)$ & $139(52)$ \\
\hline Diabetes mellitus, $\mathrm{n}(\%)$ & $126(17)$ & $99(20)$ & $27(10)$ \\
\hline Smoker (current/former), n (\%) & $365(48)$ & $265(54)$ & $100(37)$ \\
\hline Hyperlipidemia, n (\%) & $321(42)$ & $222(45)$ & $99(37)$ \\
\hline \multicolumn{4}{|l|}{ Stroke subtypes } \\
\hline Atherothrombotic, n (\%) & $109(14)$ & $80(16)$ & $29(11)$ \\
\hline Cardioembolic, n (\%) & $160(21)$ & $109(22)$ & $51(19)$ \\
\hline Lacunar, n (\%) & $191(25)$ & $133(27)$ & $58(22)$ \\
\hline Other, n (\%) & $55(7)$ & $27(5)$ & $28(10)$ \\
\hline Unknown, n (\%) & $248(33)$ & $145(29)$ & $103(38)$ \\
\hline Symptoms = 24 hours, $\mathrm{n}(\%)$ & $276(36)$ & $164(33)$ & $112(42)$ \\
\hline Previous stroke or transient ischemic attack, n (\%) & $136(18)$ & $89(18)$ & $47(18)$ \\
\hline Atrial fibrillation, n (\%) & $94(12)$ & $67(14)$ & $27(10)$ \\
\hline History of myocardial infarction, n (\%) & $62(8)$ & $52(I I)$ & $10(4)$ \\
\hline Migraine, n(\%) & $36(5)$ & $7(1)$ & $29(I I)$ \\
\hline
\end{tabular}

Female ES positive patients showed a lower prevalence of atrial fibrillation $(P<0.001)$. In male ES positive patients no significant association with atrial fibrillation was found.

The crude odds ratios confirmed that male stroke patients with RLS are at higher risk for cryptogenic strokes (OR 2.08; 95\% CI 1.37-3.14). Multiple logistic regression analysis with adjustment for age leads to a substantial decrease of the effect of the PFO (aOR 1.56; 95\% CI 1.00$2.43)$, which is no longer significant. The corresponding values for female patients showed a nonsignificant risk for cryptogenic stroke for ES-positive patients (OR 1.60; 95\% CI 0.98-2.64), an effect which completely disappeared after adjustment for age (aOR 0.97; 95\% CI 0.78-2.22).

Stroke of unknown origin in men and women was associated with younger age $(P<0.001)$.

\section{Discussion}

We found a significant association between RLS and cryptogenic stroke. This has frequently been reported in previous publications, which initially led to the consideration of PFO as an important risk factor in stroke. [4-9] Also in accordance with previous studies, ES positive patients were younger and, as expected, less likely to have traditional risk factors. A higher prevalence of PFO in young subjects has also been reported in the general population.[10,11] The association of RLS and cryptogenic stroke might therefore be coincidence. When adjusting for age, there was no longer a significant correlation between RLS and cryptogenic stroke in our study, which reduced the suggested statistical association between cryptogenic stroke and PFO. This is in line with a recently published population-based study which also describes a much weaker association between PFO and cryptogenic stroke than has been reported earlier.[12,13]

Table 2: Relative frequency of ES in different stroke subtypes

\begin{tabular}{|c|c|c|c|}
\hline Stroke Subtypes & ES-Positive during Valsalva, $\mathrm{n}(\%)$ & ES-Negative, n (\%) & Difference $P$-value \\
\hline \multicolumn{4}{|l|}{ Male patients $(n=494)$} \\
\hline Atherothrombotic & $16(20.0)$ & $64(80.0)$ & 0.078 \\
\hline Cardioembolic & $28(25.7)$ & $81(74.3)$ & 0.548 \\
\hline Lacunar & $32(24.1)$ & $101(75.9)$ & 0.217 \\
\hline Other & $7(25.9)$ & $20(74.1)$ & 1.000 \\
\hline Unknown & $57(39.3)$ & $88(60.7)$ & 0.001 \\
\hline \multicolumn{4}{|c|}{ Female patients $(n=269)$} \\
\hline Atherothrombotic & I5 (5I.7) & $14(48.3)$ & 0.322 \\
\hline Cardioembolic & $19(37.3)$ & $32(62.7)$ & 0.436 \\
\hline Lacunar & $21(36.2)$ & $37(63.8)$ & 0.298 \\
\hline Other & $8(28.6)$ & $20(71.4)$ & 0.157 \\
\hline Unknown & $51(49.5)$ & $52(50.5)$ & 0.076 \\
\hline
\end{tabular}


Table 3: ES at rest in the patients who were ES-positive during Valsalva

\begin{tabular}{llll}
\hline Stroke Subtypes & ES-Positive at Rest, $\mathrm{n}(\%)$ & ES-Negative at Rest, $\mathrm{n}(\%)$ & Difference P-value \\
\hline Male patients $(\mathrm{n}=140)$ & & & 1.000 \\
Atherothrombotic & $8(50.0)$ & $8(50.0)$ & 0.291 \\
Cardioembolic & $16(57.1)$ & $12(42.9)$ & 0.427 \\
Lacunar & $13(40.6)$ & $19(59.4)$ & 0.447 \\
Other & $2(57.1)$ & $5(42.9)$ & 1.000 \\
Unknown & $27(47.4)$ & $30(52.6)$ & 1.000 \\
& & & 0.201 \\
Female patients $(\mathrm{n}=114)$ & $6(40.0)$ & $9(60.0)$ & 1.000 \\
Atherothrombotic & $10(52.6)$ & $9(47.4)$ & $13(61.9)$ \\
Cardioembolic & $8(38.1)$ & $4(50.0)$ & 0.709 \\
Lacunar & $4(50.0)$ & $35(68.6)$ & 0.179 \\
Other & $16(31.4)$ & & \\
Unknown & & & \\
\hline
\end{tabular}

Unlike most previous studies, we stratified for gender: In female patients, who were significantly more likely to show RLS, the correlation between RLS and cryptogenic stroke did not reach significance and further decreased after adjusting for age. However, among male patients cryptogenic stroke still weakly correlated with RLS in multivariate analysis (CI 1.00-2.43). Conflicting with our results, neither the autoptic study of Hagen et al. nor the abovementioned population-based study showed the incidence of PFO to differ significantly between men and women.[11,12,14] Of both previous large multicenter studies only the PFO/ASA collaborative study reported a significant correlation of female gender and PFO.[4,15] The reasons for any gender-related differences among stroke patients remain uncertain and require further exploration.

Our results furthermore question the common theory of paradoxical embolism: Presuming arterial embolism via PFO secondary to a venous embolic source, the amount of shunt volume would be expected to correlate with the risk of stroke. This thesis has support from prior TEE-based studies. $[16,17]$ Anzola et al found that detection of more than 10 bubbles by c-TCD correlated with stroke recurrence.[18] However, the number of patients included in these studies were small, and the criteria for grading the size and thresholds for distinguishing a large shunt from a small one were arbitrary. In contrast, in both previous large TEE-based multicenter studies, PFO size failed as a significant predictor of stroke recurrence. $[4,15]$ Further studies revealed that the amount of contrast shunting did not correlate with the size of the PFO whether measured by two-dimensional TEE or invasively by balloon sizing. [19] Exploiting the method of c-TCD, we detected the shunted contrast medium directly in the target organ. Furthermore our method allowed us to discriminate between the presence of a shunt at rest versus RLS during Valsalva maneuver. Only in the first subgroup would the condition for paradoxical embolism be continuously satisfied. In the remaining patients, particular circumstances would be required that cause a transient right-to-left intracardiac shunt precisely at the moment an embolus passes the right atrium. This is less likely, and previous investigations did not reveal an association between Valsalva-provoking activities preceding stroke onset and the presence of PFO. [5, 20,21] Hence, we expected a particularly high percentage of patients with RLS at rest among patients who otherwise had no identifiable causes of stroke. However, this was not the case;

Another possible explanation for stroke secondary to PFO but independent of paradoxical embolism is secondary cardiac arrhythmias. [22] We did not find atrial fibrillation to be associated with RLS. Thus, our study does not provide support for cardiac arrhythmia as a relevant mechanism of stroke in PFO carriers. These findings are in line with previous studies.[20]

Other possible explanations include abnormalities of the endocardial surface of the septum or within the PFO that are a focus for thrombus formation.[23] A substantial limitation is that c-TCD is not applicable in detecting distinctive features like an atrial aneurysm. A further unavoidable limitation of this method is that patients with severe stroke and very old patients are probably underrepresented, as the former may not be able to perform Valsalva maneuver and in the latter group it might be difficult to perform the transcranial Doppler examination because of insufficient "bone windows."

Despite these limitations, the large number of patients included and the use of c-TCD contribute new arguments, particularly by weakening the thesis of a significant correlation between PFO and cryptogenic stroke and assessing potential gender-related differences that should be taken into account in future studies. 


\section{Competing interests}

The authors declare that they have no competing interests.

\section{Acknowledgements}

We would like to thank B. Eckenweber, C. Leege, C. Leonhard and R. Siegert for technical report and $\mathrm{M}$. Ploner and $\mathrm{O}$. Stuve for critical review of the manuscript

\section{References}

I. Adams HP Jr, Bendixen BH, Kappelle LJ, Biller J, Love BB, Gordon DL, Marsh EE 3rd: Classification of subtype of acute ischemic stroke. Definitions for use in a multicenter clinical trial. TOAST. Trial of Org 10172 in Acute Stroke Treatment. Stroke 1993, 24(I):35-4I.

2. Schwarze J], Sander D, Kukla C, Wittich I, Babikian VL, Klingelhofer J: Methodological parameters influence the detection of rightto-left shunts by contrast transcranial Doppler ultrasonography. Stroke 1999, 30(6): 1234-1239.

3. Jauss $M$, Zanette E: Detection of right-to-left shunt with ultrasound contrast agent and transcranial Doppler sonography. Cerebrovasc Dis 2000, 10(6):490-496.

4. Homma S, Sacco RL, Di Tullio MR, Sciacca RR, Mohr JP: Effect of medical treatment in stroke patients with patent foramen ovale: patent foramen ovale in Cryptogenic Stroke Study. Circulation 2002, 105(22):2625-2631.

5. Bogousslavsky J, Garazi S, Jeanrenaud X, Aebischer N, van Melle G: Stroke recurrence in patients with patent foramen ovale: the Lausanne Study. Lausanne Stroke with Paradoxal Embolism Study Group. Neurology 1996, 46(5): I 30 I- 1305.

6. Di Tullio M, Sacco RL, Gopal A, Mohr JP, Homma S: Patent foramen ovale as a risk factor for cryptogenic stroke. Ann Intern Med 1992, I I 7(6):46I-465.

7. Webster MW, Chancellor AM, Smith HJ, Swift DL, Sharpe DN, Bass NM, Glasgow GL: Patent foramen ovale in young stroke patients. Lancet 1988, 2(860 I): II-I2.

8. de Belder MA, Tourikis L, Leech G, Camm AJ: Risk of patent foramen ovale for thromboembolic events in all age groups. Am J Cardiol 1992, 69(16):1316-1320.

9. Handke M, Harloff A, Olschewski M, Hetzel A, Geibel A: Patent foramen ovale and cryptogenic stroke in older patients. $N$ Engl J Med 2007, 357(22):2262-2268.

10. Patten B: The closure of the foramen ovale. Am J Anat 193I, 48(I): 19-44.

1I. Hagen PT, Scholz DG, Edwards WD: Incidence and size of patent foramen ovale during the first 10 decades of life: an autopsy study of 965 normal hearts. Mayo Clin Proc 1984, 59(I): 17-20.

12. Petty GW, Khandheria BK, Meissner I, Whisnant JP, Rocca WA, Christianson T], Sicks JD, O'Fallon WM, McClelland RL, Wiebers DO: Population-based study of the relationship between patent foramen ovale and cerebrovascular ischemic events. Mayo Clin Proc 2006, 8I(5):602-608

13. Meissner I, Khandheria BK, Heit JA, Petty GW, Sheps SG, Schwartz GL, Whisnant JP, Wiebers DO, Covalt JL, Petterson TM, et al.: Patent foramen ovale: innocent or guilty? Evidence from a prospective population-based study. J Am Coll Cardiol 2006, 47(2):440-445.

14. Meissner I, Whisnant JP, Khandheria BK, Spittell PC, O'Fallon WM, Pascoe RD, Enriquez-Sarano M, Seward JB, Covalt JL, Sicks JD, et al.: Prevalence of potential risk factors for stroke assessed by transesophageal echocardiography and carotid ultrasonography: the SPARC study. Stroke Prevention: Assessment of Risk in a Community. Mayo Clin Proc 1999, 74(9):862-869.

15. Mas JL, Arquizan C, Lamy C, Zuber M, Cabanes L, Derumeaux G, Coste J: Recurrent cerebrovascular events associated with patent foramen ovale, atrial septal aneurysm, or both. N Engl J Med 200I, 345(24): 1740-1746.

16. Serena J, Segura T, Perez-Ayuso MJ, Bassaganyas J, Molins A, Davalos $A$ : The need to quantify right-to-left shunt in acute ischemic stroke: a case-control study. Stroke 1998, 29(7): I322-I328.

17. De Castro S, Cartoni D, Fiorelli M, Rasura M, Anzini A, Zanette EM, Beccia M, Colonnese C, Fedele F, Fieschi C, et al:: Morphological and functional characteristics of patent foramen ovale and their embolic implications. Stroke 2000, 3 I ( I 0):2407-24 I3.
18. Anzola GP, Zavarize P, Morandi E, Rozzini L, Parrinello G: Transcranial Doppler and risk of recurrence in patients with stroke and patent foramen ovale. Eur J Neurol 2003, 10(2): 129-I35.

19. Schuchlenz HW, Weihs W, Beitzke A, Stein JI, Gamillscheg A, Rehak P: Transesophageal Echocardiography for Quantifying Size of Patent Foramen Ovale in Patients With Cryptogenic Cerebrovascular Events. Stroke 2002, 33(I):293-296.

20. Lamy C, Giannesini C, Zuber M, Arquizan C, Meder JF, Trystram D, Coste J, Mas JL: Clinical and Imaging Findings in Cryptogenic Stroke Patients With and Without Patent Foramen Ovale: The PFO-ASA Study. Stroke 2002, 33(3):706-7II.

21. Ranoux D, Cohen A, Cabanes L, Amarenco P, Bousser MG, Mas JL: Patent foramen ovale: is stroke due to paradoxical embolism? Stroke 1993, 24(I):31-34.

22. Berthet K, Lavergne T, Cohen A, Guize L, Bousser MG, Le Heuzey JY, Amarenco P: Significant association of atrial vulnerability with atrial septal abnormalities in young patients with ischemic stroke of unknown cause. Stroke 2000, 3 I (2):398-403.

23. Fox ER, Picard MH, Chow CM, Levine RA, Schwamm L, Kerr AJ: Interatrial septal mobility predicts larger shunts across patent foramen ovales: an analysis with transmitral Doppler scanning. Am Heart J 2003, I45(4):730-736.

Publish with Bio Med Central and every scientist can read your work free of charge

"BioMed Central will be the most significant development for disseminating the results of biomedical research in our lifetime. "

Sir Paul Nurse, Cancer Research UK

Your research papers will be:

- available free of charge to the entire biomedical community

- peer reviewed and published immediately upon acceptance

- cited in PubMed and archived on PubMed Central

- yours - you keep the copyright 